\title{
Advanced Microcomputer-Aided Medical Illustration
}

\author{
Felix S. Chew and Martha L. Hefner
}

In a visually oriented field such as diagnostic radiology, illustrations, diagrams, and other types of artwork can complement verbal description and photographs, simplify the description of anatomic concepts, and add interest and aesthetic value to a presentation or manuscript. The vast potential of the microcomputer for creating high-quality art and graphics is being realized by the availability of high-level illustration programs running on fast computer systems. Advanced microcomputer applications for illustration have the tools to compose and execute drawings of virtually unlimited detail and complexity. Digitized images can be used as drawing templates or as components of finished art. Text is easily incorporated. Each individual element of a drawing can be changed or edited without altering other portions of the drawing. Finished art can be output to laserxerographic printers, film recorders, and photo imagesetters.

(1) 1990 by W.B. Saunders Company.

KEY WORDS: computers, computer graphics, medical illustration.

$\mathbf{I}^{\mathrm{N}}$ N A VISUALLY oriented field such as diagnostic radiology, illustrations, diagrams, and other types of artwork can complement verbal description and photographs. Artwork can be of particular value when an anatomic structure or feature is established indirectly. For example, when intraluminal contrast material is used to outline an alimentary tract mass, a drawing of the lesion from outside the lumen or in crosssection may help the reader to understand exactly what the contrast material is outlining and why the diagnostic images appear as they do. Artwork may also simplify the description of anatomic concepts and add interest and aesthetic value to a presentation.

The medical illustrator should be involved in the project from an early stage. The information and concepts to be illustrated should be ex-

From the Department of Radiology, Massachusetts General Hospital and Harvard Medical School, Boston, MA, and the Medical Illustration and Graphics Unit, Division of Educational Communications, State University of New York Health Science Center at Syracuse.

Address reprint requests to Felix $S$. Chew, MD, Department of Radiology, Massachusetts General Hospital and Harvard Medical School, Boston, MA 02114

(1) 1990 by W.B. Saunders Company.

0897-1889/90/0301-0006\$03.00/0 plained in detail to the artist using sketches, specimens, anatomic models, photographs, and so forth. The illustrator can then present the information and concepts in a visual form and create the final artwork. An initial sketch is made from which successive versions are prepared in concert with the author. When the final version is satisfactory, the sketch is "inked in," resulting in a finished piece of artwork.

The microcomputer is a tool that can be used to transform the visual concept into artwork. ${ }^{1}$ The artist, using the computer, can create straight lines, smooth curves, regular geometric shapes, and other elements of graphic design. Most important is the ability to create smooth, curved lines of irregular path and variable weight for executing elaborate drawings. Alterations can be made rapidly and with minimal effort. Although the computer will not transform an artistically maladroit physician into a professional illustrator, in the hands of a skilled medical artist, the microcomputer can rival traditional artists' techniques such as pen and ink and color and brush in producing high-quality finished artwork for medical illustration.

This article explains the use of microcomputers in the production of a series of medical illustrations for a textbook of skeletal radiology. ${ }^{2}$

\section{MATERIALS AND METHODS}

The following computer systems and peripherals were used in various combinations: Macintosh II, Macintosh SE, and Macintosh Plus computers with various hard and floppy-disk drives (Apple Computer, Cupertino, CA); Laserwriter NTX, Laserwriter Plus, and Imagewriter II printers (Apple); and DEST PC Scan Plus image scanner (digitizer) (DEST Corp, Milpitas, CA). The Macintosh computers have a mouse that can control the movements of the cursor on the monitor screen with great precision.

The software programs that were used included Illustrator 88 (Adobe Systems, Mountain View, CA), SuperPaint (Silicon Beach Software, San Diego, CA), and Cricket Draw (Cricket Software, Malvern, PA). The following descriptions of methods reflect the use of Illustrator $88^{3 \cdot 4}$ running on a Macintosh II with 5 Mbyte of random access memory.

\section{Creating the Initial Sketch}

Once the graphic concept is clear and the composition of the artwork has been planned, a preliminary rough sketch is made. This sketch may be a freehand pencil drawing, a tracing of a radiographic image, or a previously rendered 
drawing with adaptations. A digitized version of this preliminary sketch is entered into the computer by way of the image scanner, producing a bit-mapped image. This image is used as a template to prepare a line drawing. The template is traced over with a freehand drawing tool (pen tool) controlled by the mouse and then discarded, leaving only the drawing. An automatic tracing tool is also available; the computer itself prepares a line drawing of the template by tracing the edges. The utility of this automatic tracing tool depends on the intricacy of the original material and the quality of the digitized image.

Radiographic images may be digitized into the computer by use of a video camera digitizer or by scanning highcontrast photographic prints; the results from either are often unsatisfactory. A more practical method is to trace over the salient features of the radiographic image by hand, using a photograph or the original film, and then digitizing the tracing with the scanner.

It is also possible simply to draw freehand in the computer using the pen tool and other drawing tools without using a template. Such tools are actually computer subroutines for creating straight lines, regular and irregular geometric shapes, smooth curves, and other graphic elements.

\section{Modifying the Drawing}

Once the initial sketch has been compiled, it can be edited, modified, and embellished. A complex drawing is built by layering many individual graphic elements, or objects, on top of each other (Fig 1). These objects are maintained by the computer as mathematically described objects rather than as bit maps, a strategy that allows them to be modified and edited by changing their attributes and parameters. For example, a circle may be described by its radius, the location of its center, and the thickness of the line at its perimeter; straight lines have a starting point, a direction, and a length. A circle may be changed in size or location, stretched into an oval, made opaque, or shaded to appear spherical, all within the computer. A line may have any location, length, or direction; it may be changed to any thickness or weight; and it may be solid, patterned, or dashed. Even if layered on top of one another, each individual element of a drawing can be selected and changed without altering any other portion of the drawing. One can also create composite objects that can be edited and modified together. Objects may be translated, rotated, reversed left for right, inverted top for bottom, stretched, duplicated, enlarged or reduced, and distorted (Figs 2 and 3). For example, to create the shadow of an object, one might duplicate the object, give it some angular distortion, make it opaque, rotate it $90^{\circ}$, and translate it to the base of the object. The view of the drawing on the computer screen can be zoomed in or out for detail work or for examination of the overall drawing.

Text can be added to the composition in a wide variety of typefaces and styles; the text can be edited with almost the same flexibility as a word processing program. Text may also be used as design elements and formed into circles or bound along curved paths.

\section{Printing the Final Version}

Object-oriented graphics programs permit the resolution and quality of the final printed output to exceed that of the computer monitor. PostScript (Adobe Systems) is the most common computer language used by high-level art and graphics programs to describe and manage graphic elements through mathematical algorithms. Printers and other computer-output devices need a PostScript raster image processor to translate these mathematical algorithms; an output device with such a processor is said to be PostScript compatible. Such a device is necessary for high-quality output.

The final versions of all the drawings in this project were printed by a PostScript-compatible laser-xerographic printer with 300 dots-per-inch (120 dots-per-centimeter) resolution on letter-size $(8.5 \times 11$-in) 80-lb white coated stock (Vintage Velvet paper, Seneca Papers, Rochester, NY). The artwork was submitted to the publisher as $5 \times 7$-in $(12.5 \times 17.5-\mathrm{cm})$ black-and-white photographic prints. A total of 60 figure parts were prepared for publication.

\section{DISCUSSION}

Several programs for creating art are available for the Macintosh computer. These programs can be categorized into paint, draw, and illustration types. A painting program produces a bit map on the computer screen. The painting can be edited only by erasing or being painted over. The resolution of the printed output is the same as that of the monitor screen. Examples of painting programs include MacPaint II (Claris Corp, Mountain View, CA) and Superpaint (Silicon Beach Software). A drawing program produces lines, circles, and other geometric shapes whose form and attributes (line weight, fill patterns, etc) can be edited subsequent to their creation. Examples of drawing programs include Cricket Draw and MacDraw II (Claris Corp). An illustration program is one that has a variety of tools, including freehand, drawing, and often painting tools. Examples of illustration programs include Illustrator 88, FreeHand (Aldus Corp, Seattle, WA), and Canvas (Deneba Software, Miami, FL). Draw and illustration programs are object oriented and usually produce PostScript files. As these types of programs have evolved, features of drawing and painting have become integrated. The approximate price range for software programs is from $\$ 100$ to $\$ 500$. Computer-aided design programs that are intended for mechanical or architectural drawing, for which exact dimensioning and scaling are critical, may lack tools for freehand drawing, and are therefore not particularly suitable for medical illustration work.

In selecting a computer system for medical illustration, the choice is restricted to the Apple Macintosh by the availability of software. Computing speed is important because of the mathe- 


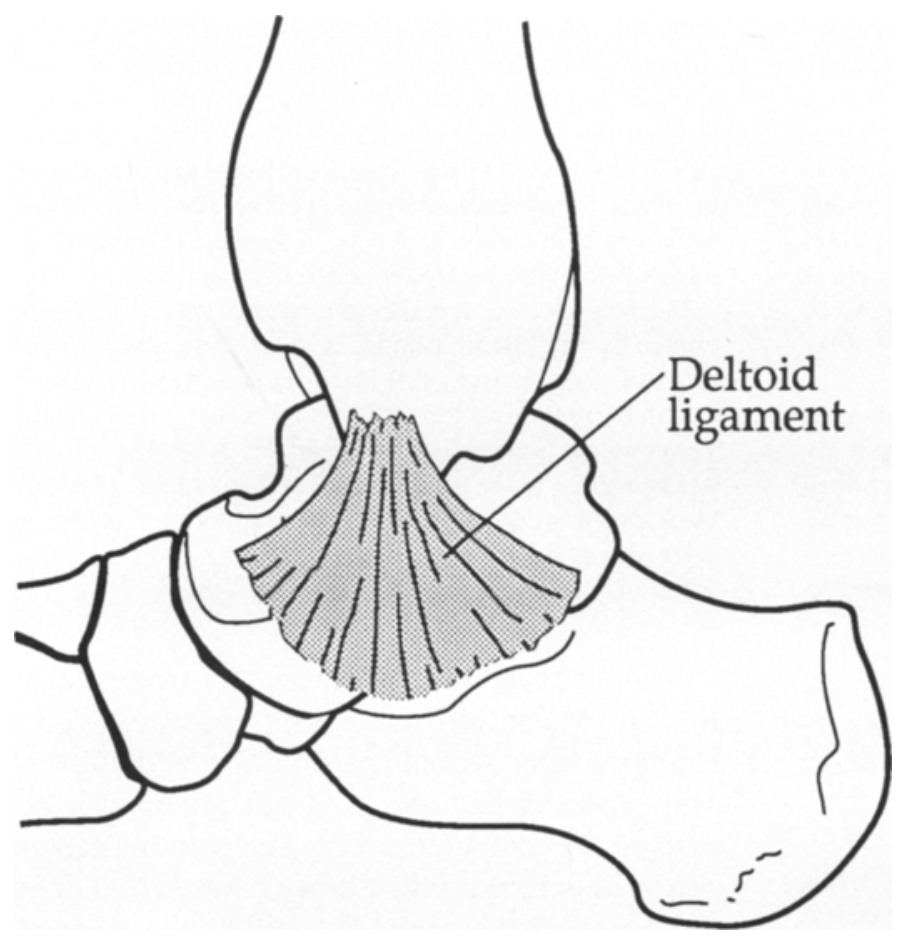

Fig 1. Deltoid ligament of the ankle (Illustrator 88 on the Macintosh II). (A) Final output printed by a PostScript-compatible laser-xerographic printer with 12 pixels $/ \mathrm{mm}$ resolution. (Reprinted from Skeletal Radiology: The Bare Bones by F. Chew with permission of Aspen Publishers, Inc, $\odot$ 1989.) (B) Final version of drawing as seen on the computer monitor. (C) (page 29) The drawing on the computer monitor as it is being worked on. The tool box on the left has options for selecting objects, moving them, 200ming the view, entering text, and so forth. Additional menus are located along the top. Scroll boxes on the right and at the bottom allow views of different portions of the drawing.

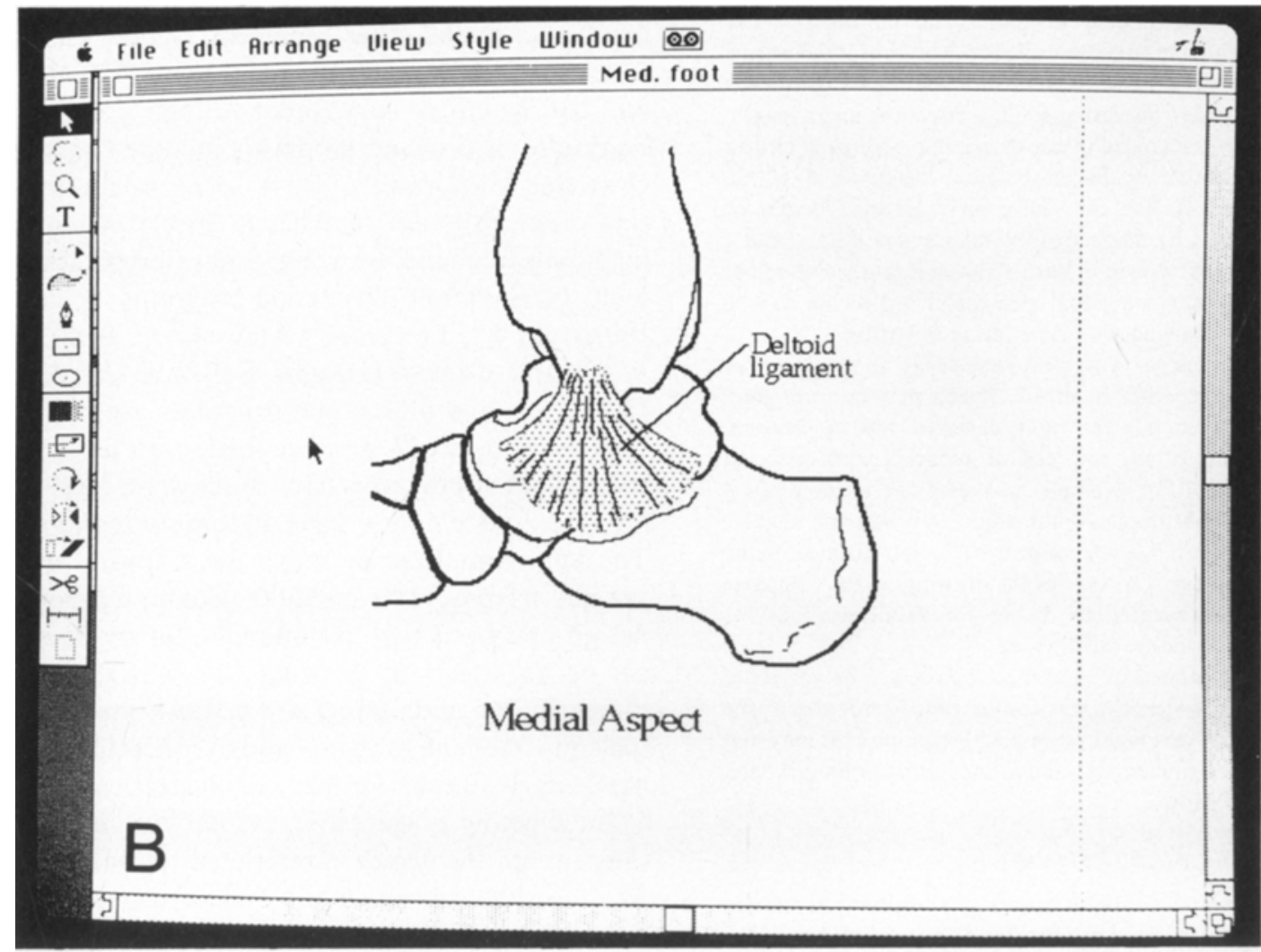




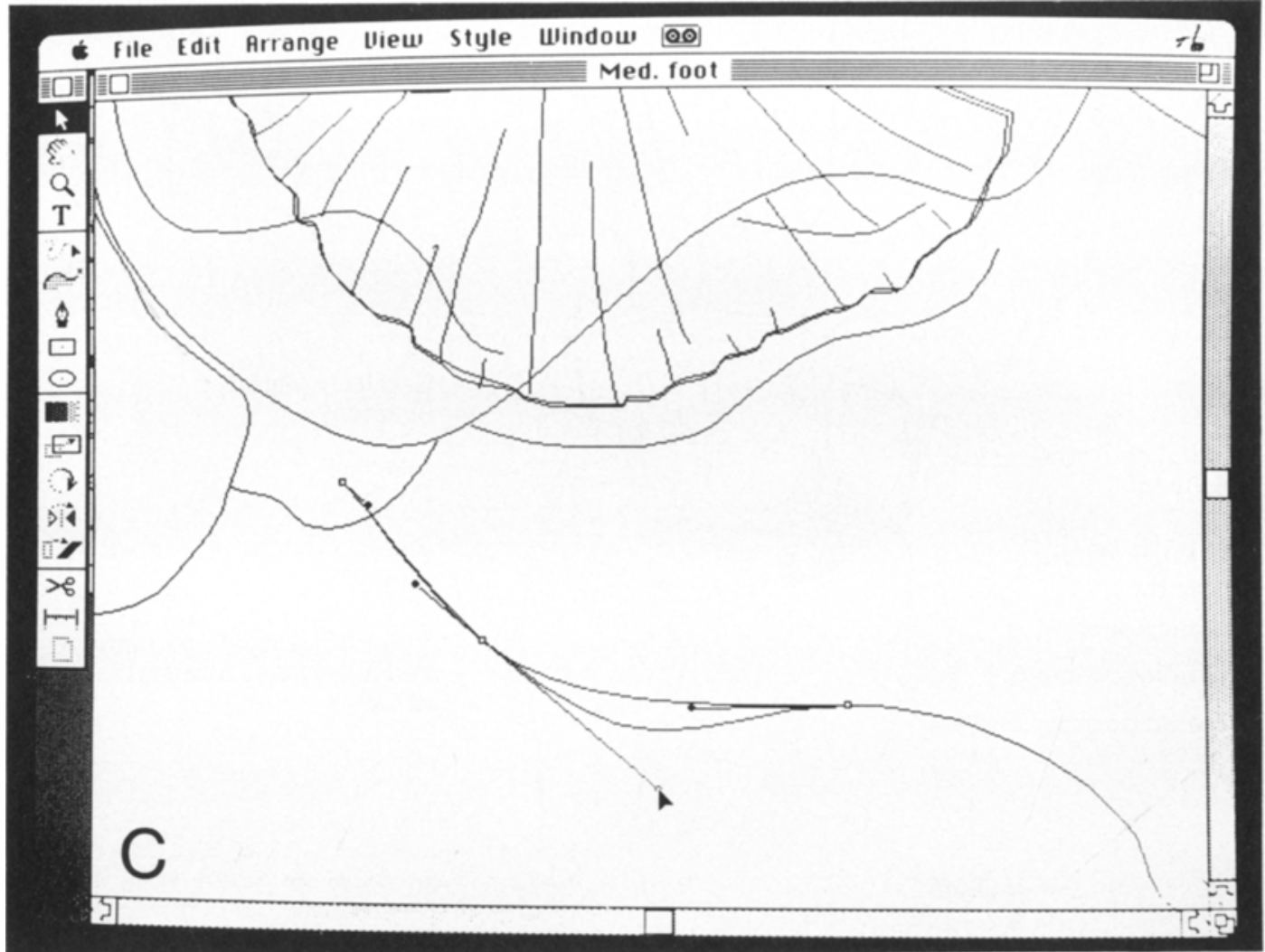

matical calculations involved each time objects are created or modified. Although the dimensions of the drawing are not limited by the size of the monitor, a larger screen size allows more of the work to be seen while drawing. The program
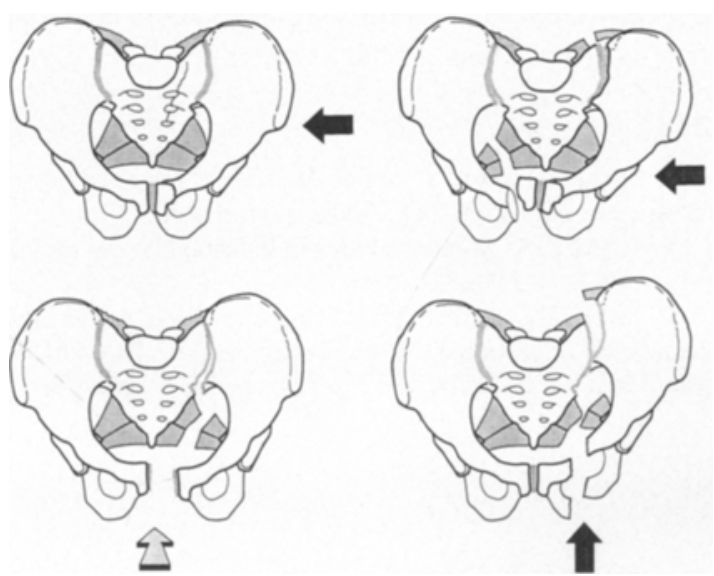

Fig 2. Pelvic fractures (lllustrator $\mathbf{8 8}$ on the Macintosh II). This series was created from a single drawing of a normal pelvis that was duplicated and fractured in different ways. (Reprinted from Skeletal Radiology: The Bare Bones by $F$. Chew with permission of Aspen Publishers, Inc, 6 1989.)
Illustrator 88 requires at least 2 Mbyte of random access memory to run properly; many Macintosh systems are supplied with only 1 Mbyte. A hard disk drive is essential because of the large size of the programs and the large size of the files they create. Mouse substitutes such as a drawing tablet with a pencillike stylus (Kurta Tablet, Kurta, Phoenix, AZ) may be helpful if one wishes to create the initial sketch by tracing over a picture. The output device of choice is a laser-xerographic printer that is Postscript compatible. A dot-matrix impact printer has much lower resolution, and although it can be used for rough drafts, it should never used to print the final artwork. Film recorders may accept graphic output for making 35-mm slides. Illustrations from the Macintosh can be output onto a photo imagesetter such as a Linotronics model L300 (Linotype $\mathrm{Co}$, Hauppauge, NY); this would allow resolution as fine as 2,540 dots per inch (100 dots per millimeter).

\section{CONCLUSION}

The vast potential of the microcomputer for creating high-quality art and graphics ${ }^{5-6}$ is being realized by the availability of high-level illustra- 


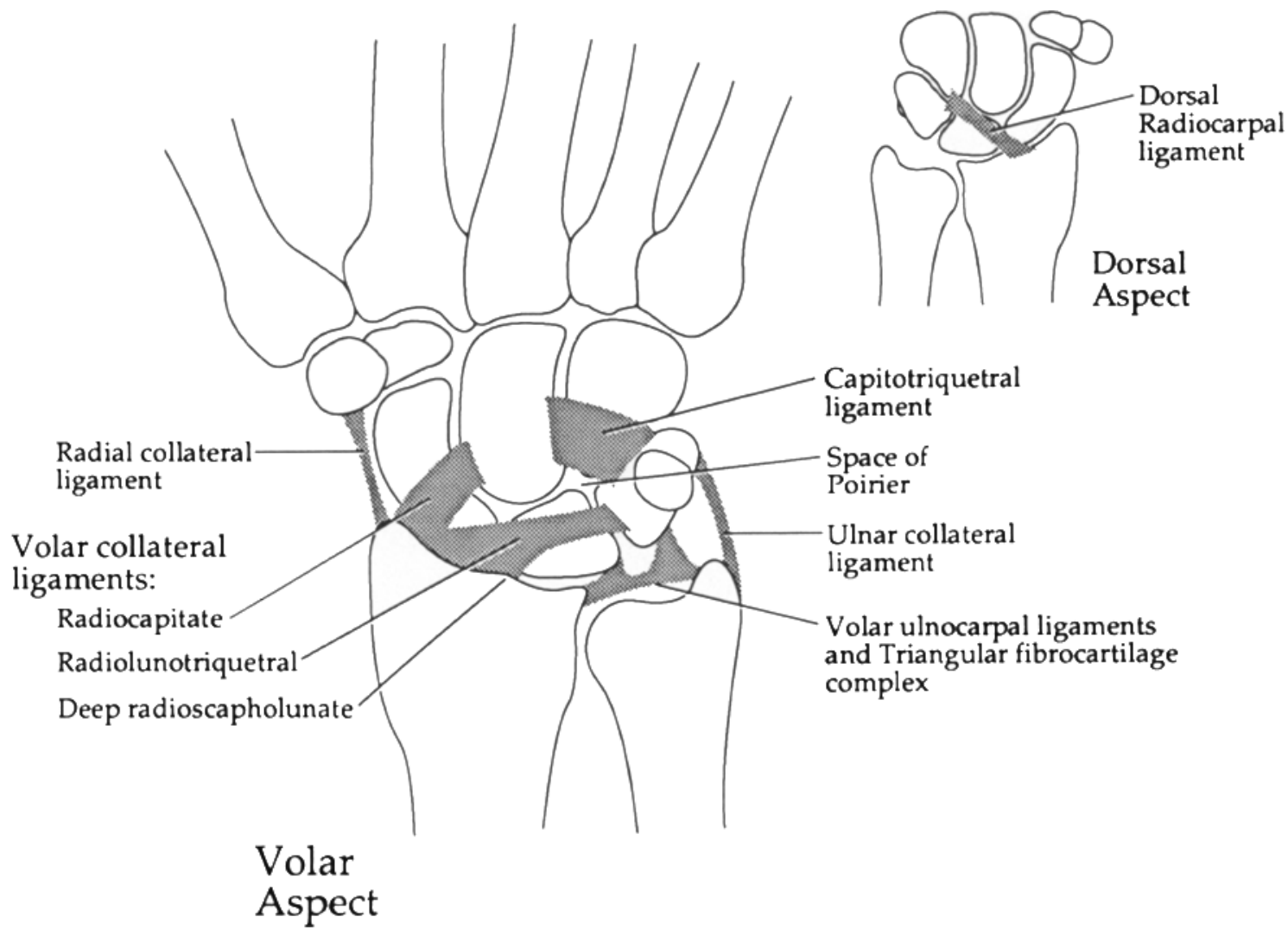

Fig 3. Ligamentous sling of the carpus (Illustrator 88 on the Macintosh II). The inset of the dorsal aspect was created by reversing the basic drawing of the bones, layering the triquetrum on top of the pisiform, and scaling the image down. The line weight and the typeface of the inset and the main drawing are uniform. (Reprinted from Skeletal Radiology: The Bare Bones by F. Chew with permission of Aspen Publishers, Inc, $\oplus$ 1989.)

tion programs running on fast computer systems. Although illustrating on the computer is still time-consuming, it is generally more efficient than using traditional artists' methods. The chief advantage of the computer is in editing, modify- ing, and changing a drawing. The computer is not a substitute for a clear conceptualization of the illustration, and it will not make a muddled concept clear. It is simply another tool the artist can use to put visual images on paper.

\section{REFERENCES}

1. Wilson S: Using computers to create art. Englewood Cliffs, NJ, Prentice-Hall, 1986

2. Chew FS: Skeletal radiology: The bare bones. Rockville, MD, Aspen, 1989

3. Bove T, Davis FE, Rhodes C: Adobe Illustrator 88: The official handbook for designers. New York, NY, Bantam, 1988
4. Burns D, Venit S, Smith D: Expert advisor: Adobe Illustrator. New York, NY, Addison-Wesley, 1988

5. Taylor GA: Microcomputer-based graphics for radiology. AJR 147:1319-1321, 1986

6. Chew FS, Hefner ML: Computer-aided design and realization of scientific exhibits in radiology. AJR 149:195198,1987 